\title{
Pyrotinib versus trastuzumab emtansine for HER2-positive metastatic breast cancer after previous trastuzumab and lapatinib treatment: a real-world study
}

\author{
Feng $\mathrm{Li}^{1,2}$, Fengrui Xu ${ }^{1,3}$, Jianbin $\mathrm{Li}^{2,4}$, Tao Wang ${ }^{2}$, Li Bian ${ }^{2}$, Shaohua Zhang ${ }^{2}$, Zefei Jiang ${ }^{2}$ \\ ${ }^{1}$ Department of Breast Oncology, Academy of Military Medical Sciences, Beijing, China; ${ }^{2}$ Department of Breast Oncology, the Fifth Medical Center \\ of Chinese PLA General Hospital, Beijing, China; ${ }^{3}$ PLA Rocket Force Characteristic Medical Center, Beijing, China; ${ }^{4}$ Department of Medical \\ Molecular Biology, Beijing Institute of Biotechnology, Academy of Military Medical Sciences, Beijing, China \\ Contributions: (I) Conception and design: Z Jiang, F Li, F Xu; (II) Administrative support: Z Jiang; (III) Provision of study materials or patients: \\ All authors; (IV) Collection and assembly of data: F Li; (V) Data analysis and interpretation: Z Jiang, F Li, F Xu; (VI) Manuscript preparation: All \\ authors; (VII) Final approval of manuscript: All authors. \\ Correspondence to: Zefei Jiang. Department of Breast Oncology, the Fifth Medical Center of Chinese PLA General Hospital, No. 8 East Street, \\ Fengtai District, Beijing 100071, China. Email: jiangzefei@csco.org.cn.
}

Background: To compare the efficacy and safety of pyrotinib and trastuzumab emtansine (T-DM1) in patients who experienced disease progression on trastuzumab and lapatinib treatment.

Methods: This was a real-world study that included cases of metastatic breast cancer (MBC) with trastuzumab and lapatinib failure. One group of patients received pyrotinib monotherapy or combination therapy, whereas the other group received T-DM1 monotherapy. The primary study endpoint was progression-free survival (PFS); secondary endpoints were the objective response rate (ORR), clinical benefit rate $(\mathrm{CBR})$ and safety.

Results: Between January 2013 and November 2019, 105 patients were enrolled in the pyrotinib group $(\mathrm{n}=55)$ or T-DM1 group ( $\mathrm{n}=50)$. The median PFS was 6.0 months (95\% CI, 4.7 to 7.3 months) with pyrotinib and 4.2 months (95\% CI, 3.6 to 4.8 months) with T-DM1 ( $\mathrm{P}=0.044$ ). ORR values were $16.3 \%$ and $20.0 \%$ in the pyrotinib and T-DM1 groups, respectively $(\mathrm{P}=0.629)$; CBR values were $45.5 \%$ and $40.0 \%$ in the pyrotinib and T-DM1 groups, respectively $(\mathrm{P}=0.573)$. Subgroup analysis of those benefitting from lapatinib revealed a median PFS of 8.1 months (95\% CI, 4.8 to 11.4 months) in the pyrotinib group, whereas that of the T-DM1 group was 4.4 months (95\% CI, 3.8 to 5.0 months, $\mathrm{P}=0.013$ ). Moreover, the median PFS of patients without liver metastases was 6.9 months (95\% CI, 3.7 to 10.1 months) in the pyrotinib group and 4.1 months (95\% CI, 3.1 to 5.1 months) in the T-DM1 group ( $\mathrm{P}=0.010)$. The main common adverse events (AEs) were diarrhea (98.2\%) and nausea (49.1\%) in the pyrotinib group and thrombocytopenia (42.0\%) and nausea $(40.0 \%)$ in the T-DM1 group. The percentages of grade 3 to 4 AEs in the pyrotinib and T-DM1 groups were $34.5 \%$ and $40.0 \%$, respectively.

Conclusions: The results of this study suggest that patients with HER2-positive MBC with trastuzumab and lapatinib failure can benefit from subsequent pyrotinib treatment and tolerate this treatment well, especially those who have benefited from previous lapatinib treatment or those who have no liver metastasis.

Keywords: Metastatic breast cancer (MBC); HER2; pyrotinib; T-DM1

Submitted May 18, 2020. Accepted for publication Oct 10, 2020.

doi: $10.21037 /$ atm-20-4054

View this article at: http://dx.doi.org/10.21037/atm-20-4054 


\section{Introduction}

The prognosis of patients with human epidermal growth factor receptor 2 (HER2)-positive metastatic breast cancer (MBC) has been significantly improved by continuous anti-HER2 targeted therapy $(1,2)$. Lapatinib is a drug recommended by the National Comprehensive Cancer Network (NCCN) and Chinese Society of Clinical Oncology for Breast Cancer (CSCO BC) guidelines after the failure of trastuzumab $(3,4)$. However, an increasing number of patients experience trastuzumab and lapatinib failure in clinical practice, and the subsequent treatment recommendations are not clearly provided by clinical guidelines (5).

Based on the availability and potential efficacy of existing drugs, subsequent options for lapatinib failure include trastuzumab emtansine (T-DM1) or the tyrosine kinase inhibitor (TKI) pyrotinib. T-DM1 is a novel antibodydrug conjugate of trastuzumab that is covalently combined with the anti-microtubule drug maytansinoid (DM1), and pyrotinib is an oral, small molecule and irreversible TKI; both are used for the treatment of HER2-positive MBC. However, only a few studies $(6,7)$ have confirmed the efficacy of T-DM1 after multiagent anti-HER2 targeted therapy, and the efficacy of pyrotinib after lapatinib failure has limited clinical verification with the exception of a case report (8). Furthermore, no head-to-head randomized controlled study has been performed to compare the efficacy of pyrotinib and T-DM1.

Against this background, we used real-world data to compare the efficacy and safety of the subsequent use of pyrotinib or T-DM1 in HER2-positive MBC after trastuzumab and lapatinib failure. We present the following article in accordance with the STROBE reporting checklist (available at http://dx.doi.org/10.21037/atm-20-4054).

\section{Methods}

\section{Study population}

In this real-world study, we enrolled patients with HER2positive MBC treated between January 2013 and September 2019 at the Department of Breast Oncology, the Fifth Medical Center of the Chinese People's Liberation Army of China (PLA) General Hospital. All these patients continued their treatment after the failure of trastuzumab and lapatinib. The inclusion criteria for patients were as follows: female, pathologically diagnosed as HER2positive [immunohistochemical $(+++)$ or fluorescent in situ hybridization detection amplification] MBC, a minimum of 50 one extracranial measurable lesion according to Response 51 Evaluation Criteria in Solid Tumors (RECIST) version 52 1.1, an Eastern Cooperative Oncology Group (ECOG) 53 performance status of 0 or 1 , and with normal liver, kidney 54 and heart function. The exclusion criteria were as follows: 55 pregnancy or breastfeeding, dyspnea, second primary malignancy or serious concomitant illness. The study was conducted in accordance with the Declaration of Helsinki (as revised in 2013). The study was approved by the Ethics Board of the affiliated hospital of Qingdao University (No. 221311920), and informed consent was obtained from all the patients.

\section{Treatment protocols}

The follow-up treatments were pyrotinib monotherapy or combination therapy and T-DM1 monotherapy, constituting the pyrotinib group and the T-DM1 group, respectively. Patients in the pyrotinib group were orally administered $400 \mathrm{mg}$ pyrotinib daily with or without other anti-tumor drugs, including cyclophosphamide, paclitaxel, albumin paclitaxel, capecitabine, etoposide or vinorelbine. Patients in the T-DM1 group received $3.6 \mathrm{mg}$ T-DM1 per kilogram of body weight every 3 weeks. The dose could be reduced and medication suspended based on the toxicity of the drug and the adverse reactions of the patients. For pyrotinib, the first dose was reduced to $320 \mathrm{mg}$ daily, compared to $3.0 \mathrm{mg}$ per kilogram for T-DM1.

\section{Efficacy assessment}

The primary endpoint was progression-free survival (PFS), which was defined as the time interval from the beginning of treatment to disease progression or any cause of death. Secondary study endpoints included the objective response rate $(\mathrm{ORR})$ and clinical benefit rate (CBR). ORR refers to the percentage of patients with a complete response (CR) and partial response (PR). CBR represents the percentage of patients with complete, partial and stable disease $\geq 6$ months as well as the safety results. The clinical efficacy of all patients was evaluated using RECIST version 1.1, and the curative effect was evaluated every 2 cycles or when the disease was judged clinically based on symptoms and signs.

\section{Safety assessment}

All adverse events (AEs) were recorded in detail, including 2 53 54 55 56 57 58 59 60 61 62 
Table 1 Characteristics of the patients at baseline

\begin{tabular}{|c|c|c|c|}
\hline Characteristic & Pyrotinib $(n=55)$ & T-DM1 $(n=50)$ & $P$ value \\
\hline Hormone-receptor status & & & 0.026 \\
\hline HR-negative & $35(63.6)$ & $21(42.0)$ & \\
\hline HR-positive & $20(36.4)$ & $29(58.0)$ & \\
\hline 1 & $8(14.5)$ & $9(18.0)$ & \\
\hline 2 & $16(29.1)$ & $13(26.0)$ & \\
\hline$\geq 3$ & $31(56.4)$ & $28(56.0)$ & \\
\hline Disease type at screening & & & 0.91 \\
\hline \multicolumn{4}{|l|}{ Metastatic site } \\
\hline Liver & $21(38.2)$ & $21(42.0)$ & 0.69 \\
\hline Lung & $30(54.5)$ & $27(54.0)$ & 0.955 \\
\hline Brain & $18(32.7)$ & $10(20.0)$ & 0.141 \\
\hline Bone & $26(47.3)$ & $23(46.0)$ & 0.896 \\
\hline Others & $39(71.0)$ & $34(68.0)$ & 0.746 \\
\hline
\end{tabular}

the description of the event and all related symptoms, time of occurrence, duration, severity, specific measures taken and final results. $\mathrm{AE}$ scores were calculated with reference to the Common Terminology Criteria for Adverse Events (CTCAE) version 4.0, and the researchers judged whether the AEs were related to pyrotinib or T-DM1.

\section{Statistical analysis}

Patients who received the different drugs were randomly assigned and analyzed. All statistical tests were performed using SPSS version 19 (SPSS Inc., Chicago, IL, USA), and all tests were two-sided with a significance level of 0.05 . For survival analysis, the Kaplan-Meier curve was used to analyze the primary endpoint of the event. The treatment differences in ORR and CBR were tested using chi-square or Fisher's exact tests.

\section{Results}

\section{Clinical characteristics}

Follow-up was performed until November 1, 2019, and a total of 105 patients with HER2-positive MBC were 121 enrolled. The median age of the subjects was 46 years old 122 (ranging from 23 to 73 years old). In total, 55 patients 123 $(52.4 \%)$ were included in the pyrotinib group, and $50 \quad 124$ patients $(47.6 \%)$ were included in the T-DM1 group. The 125 baseline demographic characteristics between the two 126 groups remained balanced (Table 1), and only the hormone 127 receptor status revealed statistically significant differences 128 (63.6\% vs. $42.0 \%, \mathrm{P}=0.026)$. Eighty-eight of these patients 129 $(83.8 \%)$ had visceral disease. There were 21 cases of liver 130 metastases both in the pyrotinib group (38.2\%) and the 131 T-DM1 group (42.0\%).

\section{Efficacy}

Of the 105 patients, 85 patients $(81.0 \%)$ achieved PFS, including 38 patients $(36.2 \%)$ in the pyrotinib group and 47 patients $(44.8 \%)$ in the T-DM1 group. Twenty patients $(19.0 \%)$ continued treatment, including 17 patients (16.2\%) in the pyrotinib group and 3 patients (2.8\%) in the T-DM1 140 group. The median PFS was 6.0 months (95\% CI, 4.7 to 141 7.3 months) in the pyrotinib group and 4.2 months $(95 \% \quad 142$ 
CI, 3.6 to 4.8 months) in the T-DM1 group $(\mathrm{P}=0.044)$ (Figure 1).

As shown in Table 2, CR was not achieved in either group. The stable disease rate was $65.5 \%$ in the pyrotinib group compared with $56.0 \%$ in the T-DM1 group, and the rate of progressive disease (PD) was $18.2 \%$ compared with $24.0 \%$, respectively. The ORR was $16.3 \%$ (9 of 55 patients) in the pyrotinib group and $20.0 \%$ (10 of 50 patients) in the T-DM1 group $(\mathrm{P}=0.629)$. The CBR was $45.5 \%$ (25 of 55 patients) in the pyrotinib group versus $40.0 \%$ (20 of 50 patients) in the T-DM1 group ( $\mathrm{P}=0.573)$. No significant differences were noted in the ORR or CBR between the two groups.

Factors for the subgroup analysis included the benefit

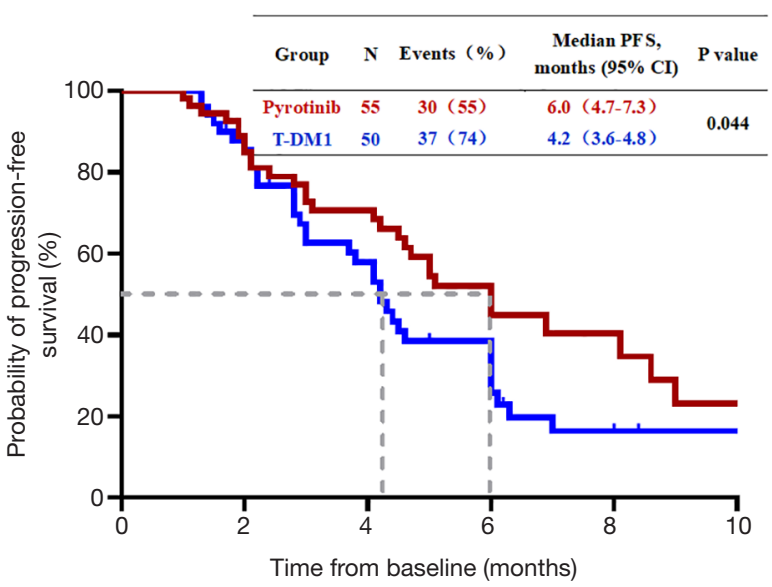

Figure 1 Kaplan-Meier estimates of progression-free survival (PFS) for all patients treated with pyrotinib and T-DM1. of prior treatment with trastuzumab or lapatinib and the 157 occurrence of liver metastases at the baseline of subsequent treatment. Among patients benefitting from lapatinib, the median PFS was 8.1 months (95\% CI, 4.8 to 11.4 months) for the pyrotinib group and 4.4 months $(95 \% \mathrm{CI}, 3.8$ to 5.0 months) for the T-DM1 group ( $\mathrm{P}=0.013$, Figure $2 A)$. The median PFS was not significantly different between patients who had benefited or not benefited from previous lapatinib treatment (Figure 2B), those who had benefited or not benefited from previous trastuzumab therapy (Figure 2C,D), those who had benefited or not benefited from the previous trastuzumab and lapatinib treatment (Figure 2E,F), and those who had liver metastases (Figure $2 G$ ). The median PFS of patients without liver metastases was 6.9 months (95\% CI, 3.7 to 10.1 months) in the pyrotinib group and 4.1 months (95\% CI, 3.1 to 5.1 months) in the T-DM1 group $(\mathrm{P}=0.010$, Figure $2 H)$.

\section{Safety}

The treatment AEs that could be tracked and recorded in either treatment group are listed in Table 3. In the pyrotinib group, the main common AEs included diarrhea $(98.2 \%)$, nausea $(49.1 \%)$, hand-foot syndrome $(40.0 \%)$, and vomiting $(38.2 \%)$; the dominating grade 3 or $4 \mathrm{AE}$ was diarrhea (21.8\%). The most frequently reported grade 3 or 4 event associated with T-DM1 was thrombocytopenia (18.0\%), and the main AEs in the T-DM1 group included thrombocytopenia $(42.0 \%)$, nausea $(40.0 \%)$, and fatigue (32.0\%). In total, 19 patients (34.5\%) and 20 patients (40.0\%) in the pyrotinib and T-DM1 groups had grade 3 to 4 AEs, 187 respectively, but no treatment-related deaths were observed.

Table 2 Comparison of efficacy between the two groups

\begin{tabular}{lccc}
\hline Type of response, No. (\%) & Pyrotinib (N=55) & T-DM1 (N=50) & P value \\
\hline Complete response & 0 & 0 & - \\
Partial response & $9(16.3)$ & $10(20.0)$ & - \\
Stable disease & $36(65.5)$ & $28(56.0)$ & - \\
SD $\geq 6$ months & $16(23.6)$ & $10(12.0)$ & - \\
Progressive disease & $10(18.2)$ & $12(24.0)$ & 0.629 \\
Objective response rate & $9(16.3)$ & $10(20.0)$ & 0.573 \\
Clinical benefit rate & $25(45.5)$ & $20(40.0)$ & \\
\hline
\end{tabular}


A

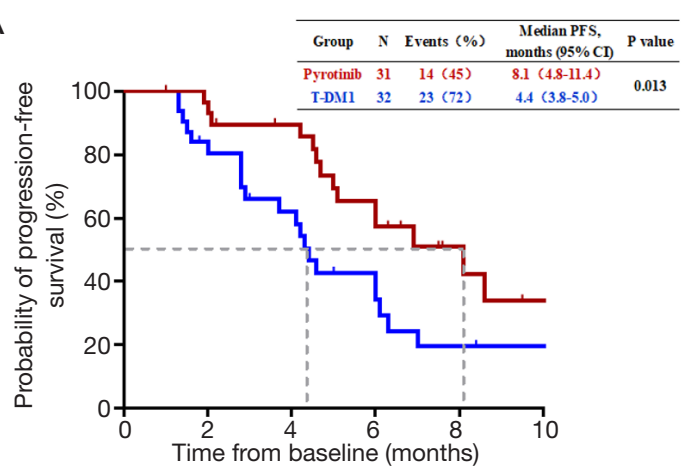

C

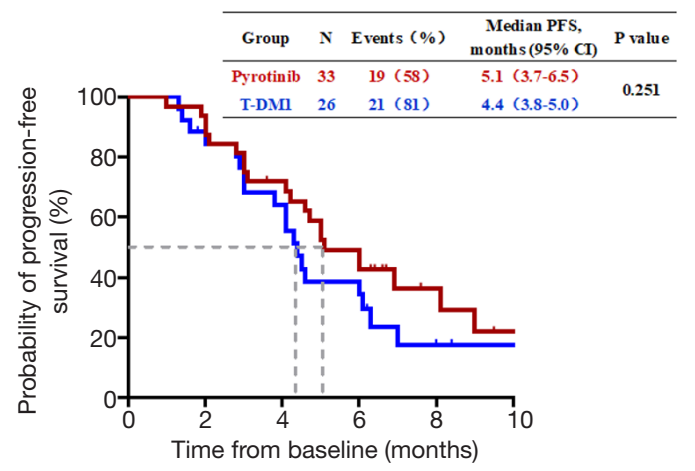

$\mathrm{E}$

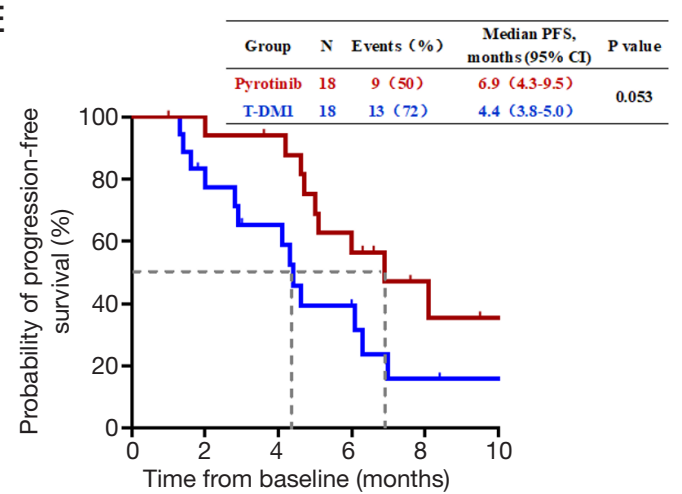

G

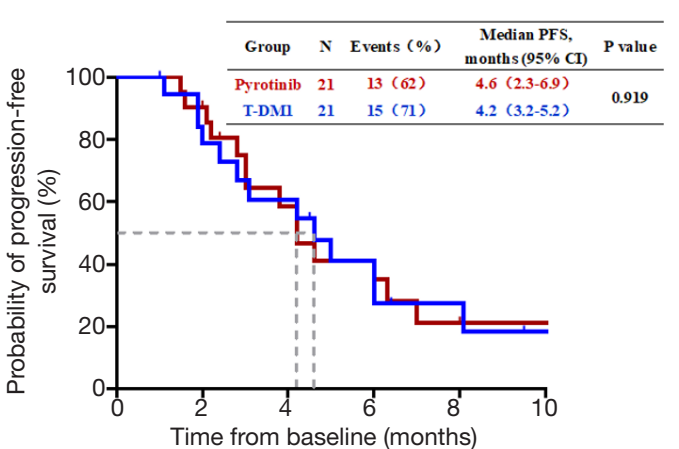

B
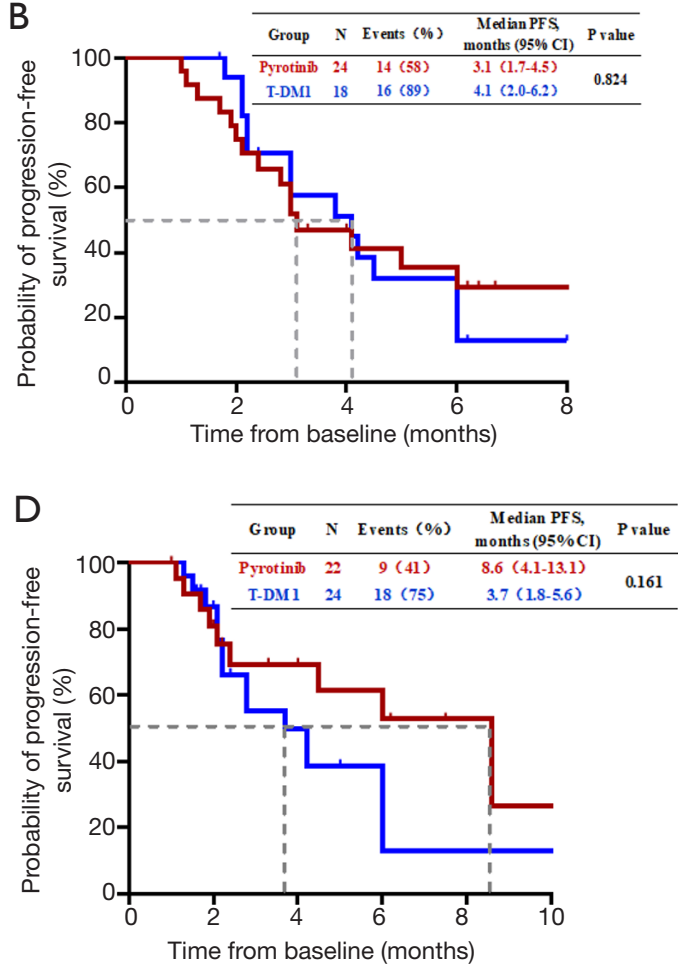

$\mathrm{F}$
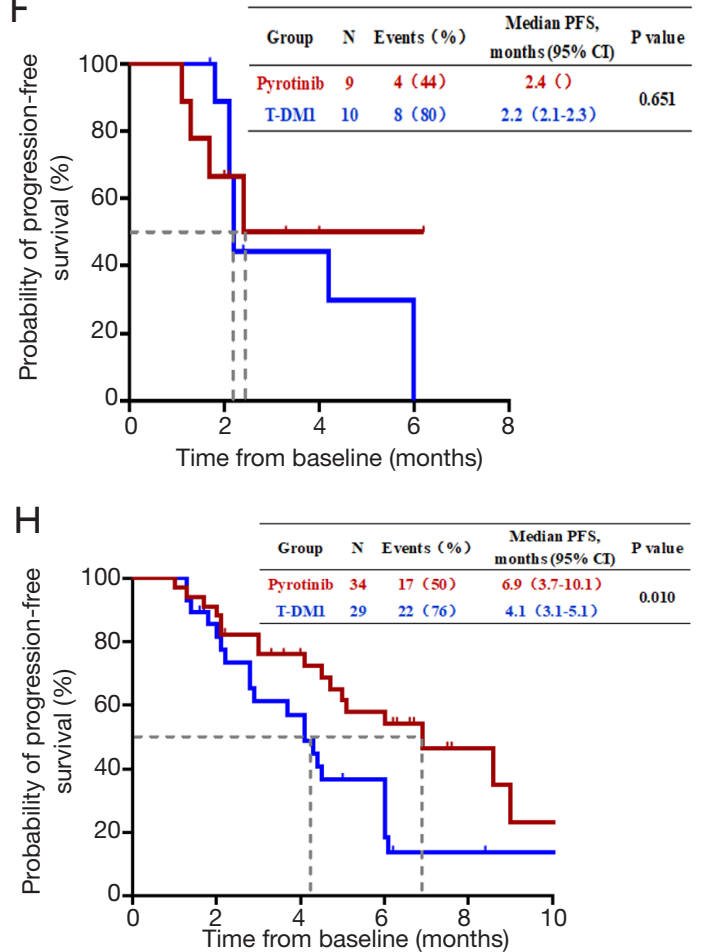

Figure 2 Kaplan-Meier estimates of PFS for the two groups. (A) Patients who have benefited from prior lapatinib; (B) patients who have not benefited from prior lapatinib; (C) patients who have benefited from prior trastuzumab; (D) patients who have not benefited from prior trastuzumab; (E) patients who have benefited from prior trastuzumab and lapatinib; (F) patients who have not benefited from prior trastuzumab and lapatinib; $(\mathrm{G})$ patients with liver metastases; $(\mathrm{H})$ patients without liver metastases. 
Table 3 Treatment-related adverse events in the two groups

\begin{tabular}{|c|c|c|c|c|}
\hline Adverse event & \multicolumn{2}{|c|}{ Pyrotinib (N=55) } & \multicolumn{2}{|c|}{ T-DM1 $(\mathrm{N}=50)$} \\
\hline Diarrhea & $54(98.2)$ & $12(21.8)$ & $5(10.0)$ & 0 \\
\hline Nausea & $27(49.1)$ & 0 & $20(40.0)$ & $3(6.0)$ \\
\hline Anemia & $23(41.8)$ & $2(3.6)$ & $6(12.0)$ & 0 \\
\hline Vomit & $21(38.2)$ & 0 & $3(6.0)$ & 0 \\
\hline Elevated transaminase & $18(32.7)$ & $1(1.8)$ & $15(30.0)$ & $2(4.0)$ \\
\hline Elevated bilirubin & $17(30.9)$ & 0 & $2(4.0)$ & 0 \\
\hline Leukopenia & $16(29.1)$ & $2(3.6)$ & $8(16.0)$ & 0 \\
\hline Fatigue & $15(27.3)$ & 0 & $16(32.0)$ & $4(8.0)$ \\
\hline
\end{tabular}

Data are No. (\%).

\section{Discussion}

Due to a lack of the availability of drugs, no studies have compared the efficacy and safety of pyrotinib with T-DM1. This study evaluated the efficacy and safety of pyrotinib and T-DM1 in HER2-positive MBC patients who received trastuzumab and lapatinib in the real world. Our results showed that the median PFS was 6.0 months in the pyrotinib group and 4.2 months in the T-DM1 group, and the ORR of the two groups was $16.3 \%$ and $20.0 \%$, respectively. The TDM4258g and TDM $4374 \mathrm{~g}$ studies $(7,9)$ explored the efficacy of T-DM1 after the failure of trastuzumab and lapatinib. The results showed that the median PFS of the two groups of patients was 4.6 months (95\% CI, 3.9 to 8.6 months) and 6.9 months (95\% CI, 4.2 to 8.4 months) respectively, and the ORR values were $25.9 \%$ and $34.5 \%$, respectively. Data from the T-DM1 group in this study, which demonstrated good efficacy of T-DM1 after the failure of trastuzumab and lapatinib, were similar to the results of the above two studies. In this study, the median PFS of the pyrotinib group was significantly better than that of the T-DM1 group, indicating that the new TKI pyrotinib may be a more valuable treatment strategy after the failure of trastuzumab and lapatinib.

Subgroup results showed that patients who had previously benefited from lapatinib and those without liver metastasis could benefit more from pyrotinib, indicating that switching to another TKI with a different mechanism may also achieve good clinical efficacy after TKI failure. 217 In addition, TKI has been used as the first-line treatment 218 for lung cancer. Patients can still benefit from another 219 TKI after the failure of first-line TKI treatment, especially 220 patients harboring the T790M mutation $(10,11)$. This study 221 demonstrated the effectiveness and safety of pyrotinib after 222 lapatinib failure, providing a reference for the dominant 223 patient population after the failure of TKI therapy. 224 However, explorations regarding the resistance mechanisms 225 of TKIs at the genetic level in the field of breast cancer remain problematic.

As noted in previous studies $(12,13)$, pyrotinib was well tolerated given that most of the AEs were grade 1 or 2 , and the main $\mathrm{AE}$ greater than grade 3 was diarrhea $(21.8 \%)$. Most of the cases of diarrhea were controllable after the medication was stopped or the dose was reduced. Similar to the TH3RESA study (6), the most common grade 1 or 2 AEs in the T-DM1 group were thrombocytopenia (42.0\%), nausea $(40.0 \%)$, and fatigue $(32.0 \%)$. No deaths related to adverse reactions occurred.

The results of this study revealed the clinical advantages of pyrotinib. However, the data were obtained from the real world and were not as rigorous as that of randomized controlled studies. In addition, long-term survival information was lacking. Therefore, clinical trial data are still required to compare the efficacy of the two drugs, and we should also assess the potential benefits of these drugs 
after the failure of TKI at the genetic level.

In conclusion, the results of this study showed that patients with HER2-positive MBC for whom trastuzumab and lapatinib failed may benefit from subsequent pyrotinib treatment and that the treatment was well tolerated, especially for patients who benefited from previous lapatinib treatment or had no liver metastasis (Research number: CSCO-BC RWS 2001).

\section{Acknowledgments}

Funding: This study was supported by Capital Clinical Characteristic Application Research (Grant number Z181100001718215).

\section{Footnote}

Reporting Checklist: The authors have completed the STROBE reporting checklist. Available at http://dx.doi. org/10.21037/atm-20-4054

Data Sharing Statement: Available at http://dx.doi. org/10.21037/atm-20-4054

Conflicts of Interest: All authors have completed the ICMJE uniform disclosure form (available at http://dx.doi. org/10.21037/atm-20-4054). The authors have no conflicts of interest to declare.

Ethics Statement: The authors are accountable for all aspects of the work in ensuring that questions related to the accuracy or integrity of any part of the work are appropriately investigated and resolved. The study was conducted in accordance with the Declaration of Helsinki (as revised in 2013). The study was approved by the Ethics Board of the affiliated hospital of Qingdao University (No. 221311920), and informed consent was obtained from all the patients.

Open Access Statement: This is an Open Access article distributed in accordance with the Creative Commons Attribution-NonCommercial-NoDerivs 4.0 International License (CC BY-NC-ND 4.0), which permits the noncommercial replication and distribution of the article with the strict proviso that no changes or edits are made and the original work is properly cited (including links to both the formal publication through the relevant DOI and the license). See: https://creativecommons.org/licenses/by-nc-nd/4.0/.

\section{References}

1. Dawood S, Broglio K, Buzdar AU, et al. Prognosis of women with metastatic breast cancer by HER2 status and trastuzumab treatment: an institutional-based review. J Clin Oncol 2010;28:92-8.

2. Perez EA, Romond EH, Suman VJ, et al. Four-year follow-up of trastuzumab plus adjuvant chemotherapy for operable human epidermal growth factor receptor 2-positive breast cancer: joint analysis of data from NCCTG N9831 and NSABP B-31. J Clin Oncol 2011;29:3366-73.

3. NCCN. Clinical practice guidelines in oncology (NCCN guidelines), breast Cancer 2020.

4. Li J, Jiang Z. CSCO BC guideline: updates for HER2 positive breast cancer in 2020. Transl Breast Cancer Res 2020;1:4.

5. Escrivá-de-Romaní $S$, Arumí M, Bellet M, et al. HER2positive breast cancer: Current and new therapeutic strategies. Breast 2018;39:80-8.

6. Krop IE, Kim SB, Martin AG, et al. Trastuzumab emtansine versus treatment of physician's choice in patients with previously treated HER2-positive metastatic breast cancer (TH3RESA): final overall survival results from a randomised open-label phase 3 trial. Lancet Oncol 2017;18:743-54.

7. Burris HA 3rd, Rugo HS, Vukelja SJ, et al. Phase II study of the antibody drug conjugate trastuzumab-DM1 for the treatment of human epidermal growth factor receptor 2 (HER2)-positive breast cancer after prior HER2-directed therapy. J Clin Oncol 2011;29:398-405.

8. Li F, Bian L, Qu Z, et al. Pyrotinib for metastatic breast cancer after multi-anti-HER2 targeted therapy: a case report. Transl Breast Cancer Res 2020;1:8.

9. Krop IE, LoRusso P, Miller KD, et al. A phase II study of trastuzumab emtansine in patients with human epidermal growth factor receptor 2-positive metastatic breast cancer who were previously treated with trastuzumab, lapatinib, an anthracycline, a taxane, and capecitabine. J Clin Oncol 2012;30:3234-41.

10. Remon J, Caramella C, Jovelet C, et al. Osimertinib benefit in EGFR-mutant NSCLC patients with T790Mmutation detected by circulating tumour DNA. Ann Oncol 2017;28:784-90.

11. Marchetti A, Palma JF, Felicioni L, et al. Early Prediction of Response to Tyrosine Kinase Inhibitors by Quantification of EGFR Mutations in Plasma of NSCLC Patients. J Thorac Oncol 2015;10:1437-43. 
12. Ma F, Ouyang Q, Li W, et al. Pyrotinib or Lapatinib 345 Combined With Capecitabine in HER2-Positive Metastatic Breast Cancer With Prior Taxanes, Anthracyclines, and/or Trastuzumab: A Randomized, Phase II Study. J Clin Oncol 2019;37:2610-9.

13. Yan M, Bian L, Hu X, et al. Pyrotinib plus capecitabine for human epidermal growth factor receptor 2-positive metastatic breast cancer after trastuzumab and taxanes (PHENIX): a randomized, double-blind, placebocontrolled phase 3 study. Transl Breast Cancer Res 2020;1:13.

Cite this article as: Li F, Xu F, Li J, Wang T, Bian L, Zhang S, Jiang $Z$. Pyrotinib versus trastuzumab emtansine for HER2-positive metastatic breast cancer after previous trastuzumab and lapatinib treatment: a real-world study. Ann Transl Med 2021;9(2):103. doi: 10.21037/atm-20-4054 